\title{
Development of Manufacturing Principle of Porous Iron by Carbothermic Reduction of Composite of Hematite and Biomass Char
}

\author{
Taichi Murakami, Takayuki Takahashi*1, Shoko Fuji², Daisuke Maruoka and Eiki Kasai \\ Graduate School of Environmental Studies, Tohoku University, Sendai 980-8579, Japan
}

Iron-based porous metals have several advantages such as high strength and low cost compared with aluminum alloy foams. In recent years, a number of manufacturing technologies for iron-based porous metals have been proposed. However, a practical process could not be developed because of lower porosity, higher cost of raw materials, and so on. In this study, a new manufacturing principle of porous iron was proposed by applying carbothermic reduction using a composite of hematite and carbonaceous material. In the reduction experiment, graphite, coal, and three different charcoals were used as reductants. Based on the results, the characteristics and mechanism of the volume change of the composite were investigated under different pressures.

Reduction behavior significantly varied among the different types of reductants. The composites containing graphite and coal shrank during the reduction process when heated up to $1373 \mathrm{~K}$. However, swelling behavior was observed for the composite prepared using charcoal containing some amounts of sodium and sulfur as ash components. When this composite was heated up to $1273 \mathrm{~K}^{2}$ a rate of $0.167 \mathrm{~K} \mathrm{~s}^{-1}$ at $0.3 \mathrm{MPa}$, porous iron with a maximum porosity of $97.7 \%$ was successfully obtained. The bulk density of the composite changed during the reduction of wustite to metallic iron and formed iron whiskers. The formation of the whiskered iron texture led to a considerable swelling of the composite. On the other hand, the composite prepared using charcoal with low sodium and sulfur concentrations in the ash did not swell after the formation of metallic iron. Furthermore, when such elements in the charcoal were preliminarily removed, the composite did not swell. [doi:10.2320/matertrans.M2017232]

(Received July 24, 2017; Accepted October 2, 2017; Published November 10, 2017)

Keywords: porous iron, carbothermic reduction, swelling, iron whisker

\section{Introduction}

Porous metals have a high potential to be used in a wide variety of applications such as ultra-lightweight structural components, heat insulation, and energy absorption ${ }^{1)}$, because these materials have unique physical and chemical properties. Porous aluminum is one of the well-known foamed metals with porosity greater than $90 \%{ }^{2)}$. However, porous iron and steel are superior to porous aluminum because of low cost, high strength, better weldability, etc.

Several methods to produce porous iron have been described $^{3)}$. Park et al. reported a production method of porous iron using the powders of cast iron $(\mathrm{Fe}-2.5$ mass $\% \mathrm{C}$ ) and a foaming agent of $\mathrm{MgCO}_{3}$ and $\mathrm{SrCO}_{3}{ }^{4)}$. The porosity of their porous iron was less than $60 \%$. Furthermore, one of the authors reported the method using the powders of iron, graphite, and iron oxide ${ }^{5)}$. In this case, the foaming gas was generated by the reduction reaction of iron oxide with graphite. However, its porosity was also less than $70 \%$. Lotus-type porous iron, in which the pores are formed by the gas generated owing to the difference in the gas solubility between the liquid and solid aligned in one direction by unidirectional solidification, was reported ${ }^{1)}$. It had higher strength than the conventional porous metals. However, the maximum porosity of the obtained porous irons was approximately $60 \%$.

A production method to obtain porous iron with porosity higher than $90 \%$ was reported using SUS powder, hexane as the foaming agent, a surface-active agent, and polyvinyl alcohol $^{6)}$. The maximum porosity obtained using this method was $98 \%$ with a pore size of $0.4-0.9 \mathrm{~mm}$. Furthermore, it is reported that the additive manufacturing method can pro-

\footnotetext{
${ }^{* 1}$ Graduate Student, Tohoku University

${ }^{* 2}$ Graduate Student, Tohoku University. Present address: Dai Nippon Printing Co., Ltd., Hakusan 924-0838, Japan
}

duce porous stainless steel ${ }^{7)}$. However, the cost of the raw materials for these processes is extremely high. Therefore, a production method with not only high porosity but also low cost is required. In light of the above-mentioned observations, the authors suggest a new method for producing iron foam using the well-mixed powders of iron oxide and carbonaceous material. During the heating of these powders, reduction of iron oxide by solid carbonaceous material starts, and $\mathrm{CO}_{2}$ gas is generated, as described in eq. (1). The generated $\mathrm{CO}_{2}$ gas reacts with solid carbonaceous material and $\mathrm{CO}$ gas is generated, as described in eq. (2). CO gas then reduces iron oxide as shown in eqs. (3)-(5). These reactions occur as chain reactions, and this process is known as carbothermic reduction. The microstructure of the formed metallic iron in the reduced composite is porous ${ }^{8)}$.

$$
\begin{gathered}
6 \mathrm{Fe}_{2} \mathrm{O}_{3}+\mathrm{C}=4 \mathrm{Fe}_{3} \mathrm{O}_{4}+\mathrm{CO}_{2} \\
\mathrm{CO}_{2}+\mathrm{C}=2 \mathrm{CO} \\
3 \mathrm{Fe}_{2} \mathrm{O}_{3}+\mathrm{CO}=2 \mathrm{Fe}_{3} \mathrm{O}_{4}+\mathrm{CO}_{2} \\
\mathrm{Fe}_{3} \mathrm{O}_{4}+\mathrm{CO}=3 \mathrm{FeO}+\mathrm{CO}_{2} \\
\mathrm{FeO}+\mathrm{CO}=\mathrm{Fe}+\mathrm{CO}_{2}
\end{gathered}
$$

When certain types of iron ore pellets were reduced by a $\mathrm{CO}$-containing gas at approximately $1173 \mathrm{~K}$, the pellets swelled fourfold compared with their initial volume ${ }^{9)}$. The formed metallic iron was whisker-shaped. This swelling of the pellets caused a serious problem in the operation of the blast furnace for iron making. Therefore, many studies have investigated methods to prevent this phenomenon. At the temperature range of 1173-1273 K, the abnormal swelling proceeds by $\mathrm{CO}$ gas reduction; however, $\mathrm{H}_{2}$ gas reduction did not lead to this swelling ${ }^{10)}$. However, there is no report 
on the production of porous iron based on the swelling phenomenon of the iron ore pellet. In this study, a possible production principle of porous iron combined with this abnormal swelling and carbothermic reduction of the composite was studied.

\section{Experimental}

\subsection{Sample preparation}

Hematite (average particle size: $0.5 \mu \mathrm{m}$, purity: $99.9 \%$ ) was used in this study. Three types of biomass chars (Charcoal A, B, and C), coal, and graphite (average size: $20 \mu \mathrm{m})$ were used as reductants. Biomass char and coal were pulverized and sieved to obtain a controlled particle size of $53-150 \mu \mathrm{m}$. The results of proximate analysis, which indicate the amount of fixed carbon, volatile matter, and ash in these carbonaceous materials, are shown in Table 1. The composition of the ash in charcoal and coal is also shown in Table 2. CA-800, shown in this table, is a sample of charcoal A heat-treated under an inert gas atmosphere at $1073 \mathrm{~K}$ for $3.6 \mathrm{ks}$. Charcoal A was deashed by washing with hydrochloric and hydrofluoric acid solutions, and CA' was obtained. Furthermore, CA'-Na was also prepared by mixing CA' with aqueous sodium hydroxide to control the concentration of $\mathrm{Na}$ with the same as ash in $\mathrm{CA}$, followed by drying at $378 \mathrm{~K}$. The gasification test of carbonaceous materials was carried out by thermogravimetric analysis under a $\mathrm{CO}_{2}$ gas flow of $3.33 \times 10^{-6} \mathrm{Nm}^{3} \mathrm{~s}^{-1}$ at a heating rate of $0.167 \mathrm{~K} \mathrm{~s}^{-1}$.

Hematite and carbonaceous materials were mixed well in a molar ratio of fixed carbon in coal to oxygen in iron oxide, $\mathrm{C} / \mathrm{O}$, of 0.8 . The mixed powder was press-shaped to obtain a columnar composite sample of $10 \mathrm{~mm}$ height. The diameter of the composite obtained using charcoal was $18 \mathrm{~mm}$, and that of the graphite and coal composite was $10 \mathrm{~mm}$. The density of the composites was different for each of the samples. For example, that of CA was $1.5 \mathrm{~g} \mathrm{~cm}^{-3}$.

\subsection{Reduction and foaming experiment}

A composite sample was heated to the target temperature

Table 1 Proximate analysis of carbonaceous materials. (mass\%)

\begin{tabular}{lccc}
\hline & F.C. & V.M. & Ash \\
\hline Graphite & 98.0 & - & 2.0 \\
\hline Coal & 55.5 & 36.1 & 8.4 \\
\hline Charcoal A [CA] & 62.4 & 35.6 & 2.0 \\
\hline Charcoal A [CA-800] & 85.3 & 8.4 & 6.3 \\
\hline Charcoal A' [CA'] & 62.3 & 37.7 & 0.0 \\
\hline Charcoal B [CB] & 91.7 & 7.3 & 1.0 \\
\hline Charcoal C [CC] & 72.3 & 15.7 & 12.0 \\
\hline
\end{tabular}

under an $\mathrm{Ar}-5 \% \mathrm{~N}_{2}$ gas flow and at different total pressures (0.1-1.0 MPa) at a heating rate of $0.167 \mathrm{Ks}^{-1}$, followed by cooling. In this study, different experimental apparatuses detailed in previous reports ${ }^{11,12)}$ were used to conduct the experiments under high and atmospheric pressures. In both experiments, the experimental conditions were the same, except for the gas flow rate. The gas flow rate for the experiment conducted under the high pressure was $3.3 \times$ $10^{-5} \mathrm{Nm}^{3} \mathrm{~s}^{-111)}$, while that for the experiment conducted at the atmospheric pressure was $8.33 \times 10^{-6} \mathrm{Nm}^{3} \mathrm{~s}^{-1}{ }^{12}$ ). The concentration of $\mathrm{CO}, \mathrm{CO}_{2}, \mathrm{H}_{2}, \mathrm{H}_{2} \mathrm{O}, \mathrm{CH}_{4}$, and $\mathrm{N}_{2}$ in the outlet gas was measured every $90 \mathrm{~s}$ by a gas chromatography. Using the amount of the generated gas, reduction degree of the composite sample could be calculated from the following equation.

$R D(T)=\frac{\int_{T_{0}}^{T} n_{\text {comp }} d T-\int_{T_{0}}^{T} n_{\text {ore }} d T-\int_{T_{0}}^{T} n_{\text {coal }} d T}{n_{\text {oxide }}} \times 100$

Here, $\mathrm{n}_{\text {comp }}$ and $\mathrm{n}_{\text {coal }}$ are the molar amounts of oxygen atom in the $\mathrm{CO}, \mathrm{CO}_{2}$ and $\mathrm{H}_{2} \mathrm{O}$ gases generated from the composite and that form coal, respectively, $\mathrm{n}_{\text {ore }}$ and $\mathrm{n}_{\text {oxide }}$ are that of oxygen atom in $\mathrm{H}_{2} \mathrm{O}$ generated from the ore and that originally contained in iron oxide in the composite, respectively. $\mathrm{T}_{\mathrm{O}}$ is the initial experimental temperature. Density of the obtained sample before and after heating was measured by Archimedes' method. The appearance and the cross section of the samples were observed. The specific surface area of the obtained sample was measured by the BET method.

\section{Results and Discussion}

\subsection{Gasification behavior of carbonaceous materials}

Figure 1 shows the weight loss fraction of carbonaceous materials heated to $1473 \mathrm{~K}$ at a heating rate of $0.167 \mathrm{~K} \mathrm{~s}^{-1}$ under a $\mathrm{CO}_{2}$ atmosphere. The rate of this fraction can also be seen in this figure. The weight loss fraction was defined using the following equation,

$$
(\text { Weight loss fraction })=\frac{W_{\text {after }}-W_{\text {before }}}{\text { F.C. }+ \text { V.M. }+ \text { Oxygen }} \times 100
$$

where $W_{\text {before }}$ and $W_{\text {after }}$ are the weight of sample before and after experiment, respectively. F.C. and V.M. are the amount of fixed carbon and volatile matter, respectively in carbonaceous materials. "Oxygen" is the oxygen amount originated from iron oxide. The weight loss occurs because of the decomposition of the volatile matter and the gasification reaction of the carbonaceous materials with the $\mathrm{CO}_{2}$ gas, as described in eq. (2). Except for graphite, the weight loss occurs at temperatures as low as $373 \mathrm{~K}$. This weight loss can be ascribed to the vaporization of free water presented in the ma-

Table 2 Composition of ash in charcoal and coal samples. (mass\%)

\begin{tabular}{|c|c|c|c|c|c|c|c|c|c|}
\hline & $\mathrm{CaO}$ & $\mathrm{Al}_{2} \mathrm{O}_{3}$ & $\mathrm{SiO}_{2}$ & $\mathrm{MgO}$ & $\mathrm{Fe}_{2} \mathrm{O}_{3}$ & $\mathrm{P}$ & $\mathrm{Na}$ & $\mathrm{K}$ & $\mathrm{S}$ \\
\hline Charcoal A [CA] & 62.26 & - & - & 8.62 & - & 1.1 & 1.6 & 3.5 & 2.1 \\
\hline Charcoal B [CB] & 68.27 & 4.16 & 2.78 & 13.26 & - & 1.6 & 0.4 & 2.6 & 0.6 \\
\hline Charcoal C [CC] & 20.57 & 5.67 & 29.74 & 3.65 & 22.73 & 1.3 & 0.9 & 4.4 & 0.7 \\
\hline Coal & 3.30 & 23.43 & 35.63 & 0.55 & 3.60 & 0.49 & 0.34 & 3.25 & 0.31 \\
\hline
\end{tabular}


terial. In addition, this fraction decreases above approximately $673 \mathrm{~K}$ because of the decomposition of the volatile matter. In contrast, no weight loss is observed for graphite below $1073 \mathrm{~K}$ because of the small amount of volatile matter presented in graphite. Above $1073 \mathrm{~K}, \mathrm{CA}$ and CB show similar gasification behavior and low gasification temperature. $\mathrm{CC}$ has a slightly higher gasification temperature than both $\mathrm{CA}$ and $\mathrm{CB}$.

\subsection{Reduction behavior of composite}

Figure 2 shows the change in reduction degree, under atmospheric pressure, of the composite samples containing $\mathrm{CA}$, graphite, and coal with temperature. Reduction degree of the composite sample containing graphite starts to increase at approximately $1000 \mathrm{~K}$. This increase plateaus at approximately $1150 \mathrm{~K}$. At this temperature, reduction degree is $11.1 \%$. It is indicative of the complete reduction of $\mathrm{Fe}_{2} \mathrm{O}_{3}$ to $\mathrm{Fe}_{3} \mathrm{O}_{4}$, assuming that the reaction in the sample is uniform. This temperature is lower than that for the gasification of graphite. This is because the direct reduction reaction described in eq. (1) proceeds in the composite sample at $1150 \mathrm{~K}^{13}$. At $1223 \mathrm{~K}$, the reduction degree increases again and the reduction reaction is completed at approximately $1350 \mathrm{~K}$. In contrast, the reduction degree of the composite containing coal and charcoal starts to increase at approximately $920 \mathrm{~K}$, and it increases with increasing temperature. This is the effect of the volatile matter because hydrogen and hydrocarbon gases are generated from the carbonaceous materials, which are the reducing agents ${ }^{12)}$. At $1373 \mathrm{~K}$, the reduction degree of all samples reaches over $90 \%$.

\subsection{Effect of carbonaceous materials on swelling under atmospheric pressure}

Figure 3 shows the appearances of the composite sample containing graphite, coal, and charcoal A (CA) before and after heating to $1373 \mathrm{~K}$ under atmospheric pressure. Reduction degree and density of the composite are also listed in this figure. The composite containing graphite and coal constricts after the experiment and the density increases. This may be because the reduced iron was sintered when heated since reduction degree is $90 \%$. In contrast, the density of the sample containing CA decreases. The porosity of the heated composite is calculated as $92.2 \%$ assuming that reduction reaction completes and the carbonaceous material is gasified completely. The abnormal swelling occurs when the reduction reaction of the iron oxide proceeds at the temperature range of $1173-1373 \mathrm{~K}$, as described above ${ }^{10)}$. Furthermore, CA composite expands in a vertical direction which is same as press-shaped direction. However, it is difficult to make clear this reason because many reactions proceeds in the composite and complex microstructure change occurs. For the graphite composite, the reduction reaction proceeds above $1373 \mathrm{~K}$ as shown in Fig. 2. Reduction degree of the coal composite at $1373 \mathrm{~K}$ is approximately $50 \%$, which is much lower than that of the CA composite. It means that most of the metallic iron in the coal composite forms above $1373 \mathrm{~K}$ and explains why the composite sample does not swell.

The change in the density of the CA composite with temperature is shown in Fig. 4. The density of the CA composite heated under atmospheric pressure of $0.1 \mathrm{MPa}$ at $1223 \mathrm{~K}$ is higher than that of $\mathrm{CA}$ before the experiment. It indicates that the effect of sintering the particles in the composite is stronger than the total effects of the decomposition of the volatile matter, the gasification of the fixed carbon, and the reduction of the iron oxide, resulting in an increasing den-

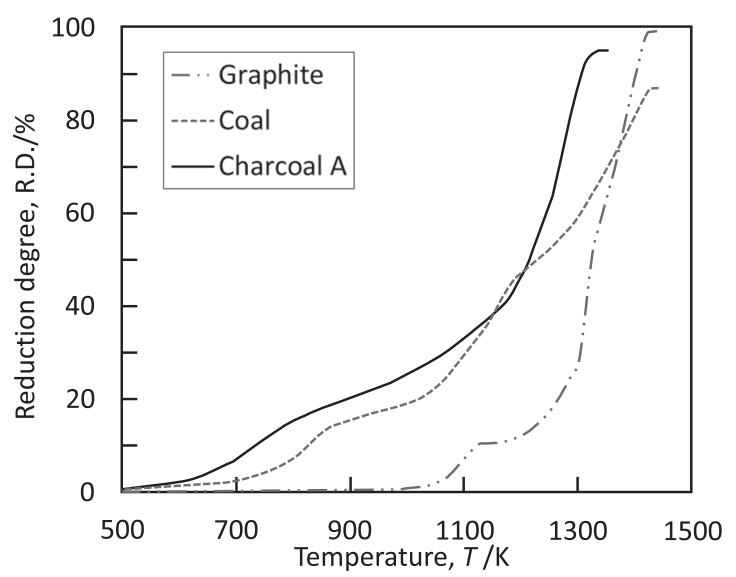

Fig. 2 Changes in reduction degree of samples observed using graphite, coal, and charcoal with temperature. (a)

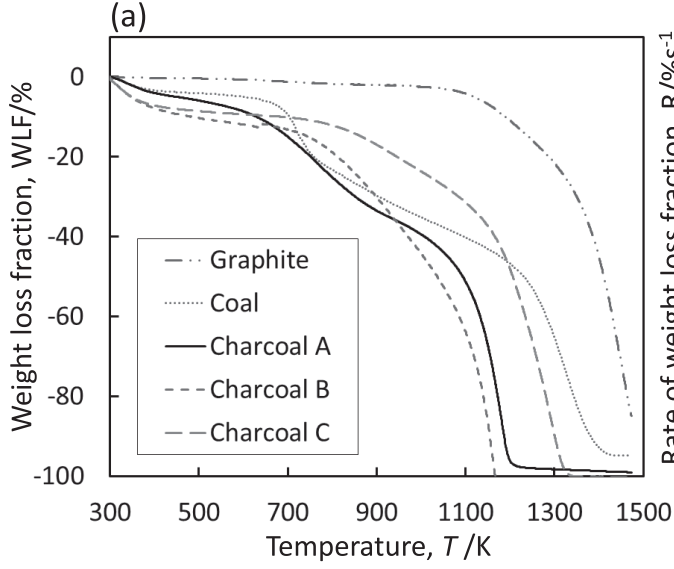

(b)

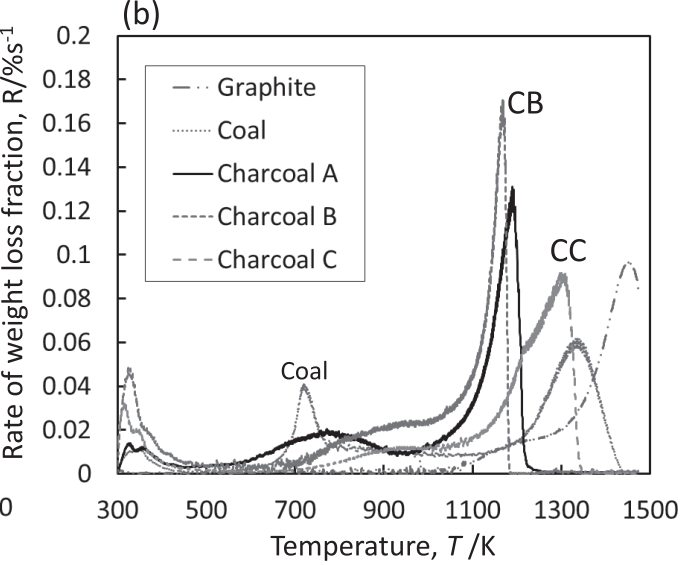

Fig. 1 Changes in (a) weigh loss fraction and (b) its rate obtained for carbonaceous materials heated up to $1473 \mathrm{~K}$ with the heating rate of $0.167 \mathrm{Ks}{ }^{-1}$ under $\mathrm{CO}_{2}$ gas atmosphere. 


\begin{tabular}{|c|c|c|c|}
\hline \multirow{2}{*}{ Before } & Graphite & Coal & CA \\
\hline & & & \\
\hline Density $\left(\mathrm{gcm}^{-3}\right)$ & 2.74 & 2.42 & 1.38 \\
\hline After & & & \\
\hline Reduction degree (\%) & 90 & & 9.3 \\
\hline Density $\left(\mathrm{gcm}^{-3}\right)$ & 3.45 & & 9.40 \\
\hline
\end{tabular}

Fig. 3 Sample appearances and values of reduction degree and density observed for composites using graphite, coal, and charcoal (CA) before and after heating up to $1373 \mathrm{~K}$ under atmospheric pressure.

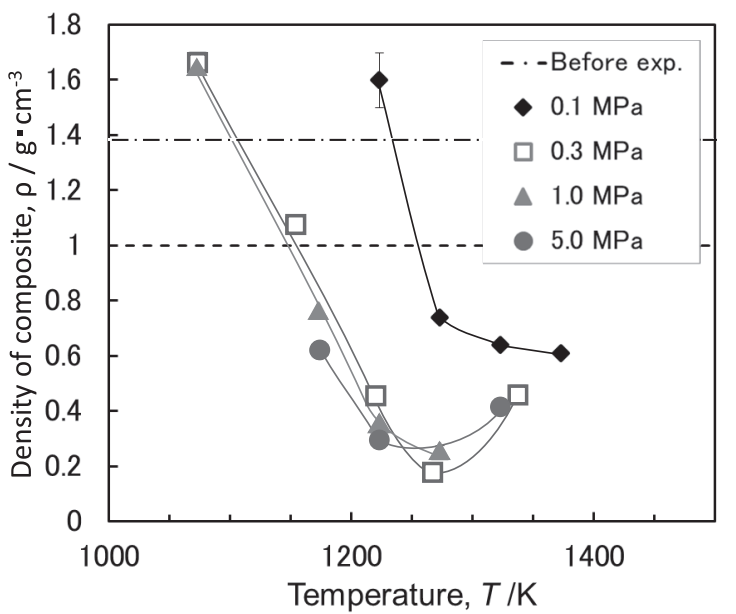

Fig. 4 Changes in density of CA composite with temperature under different total pressure.

sity. On the other hand, the density rapidly decreases when the temperature is increased above $1223 \mathrm{~K}$. Assuming that carbon and oxygen in the composite react and gasify, and the volume of the composite does not change during the experiment, the density of the composite after the experiment is calculated as $0.87 \mathrm{gcm}^{-3}$. However, at $1373 \mathrm{~K}$ the density is $0.6 \mathrm{gcm}^{-3}$, which is lower than the calculated value. It shows that this density change is caused by not only the effect of the chemical reaction but also by other reasons such as the abnormal swelling described above.

\subsection{Effect of pressure on reaction and density of composite}

Figure 5 shows the weight loss fraction of CA composite with temperature under different pressures. Here, the weight loss fraction is regarded as $100 \%$ when the decomposition of the volatile matter, the reduction of the iron oxide, and the gasification of the fixed carbon in the charcoal are com-

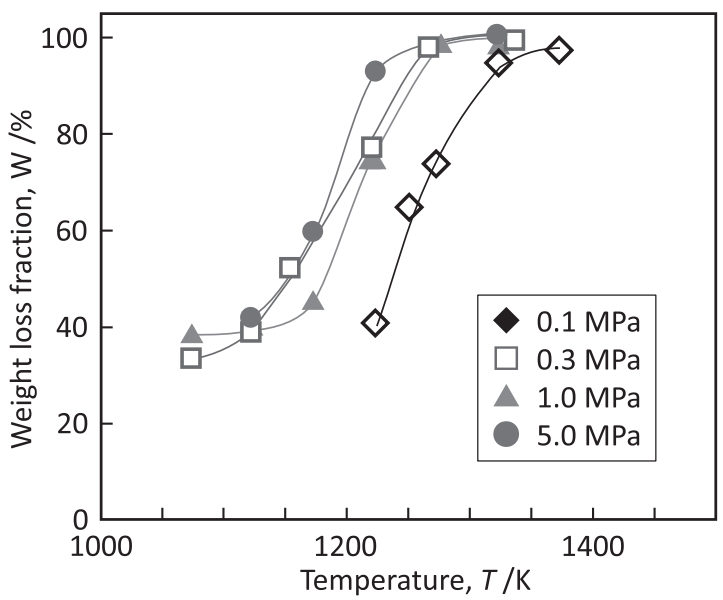

Fig. 5 Changes in weight loss fraction of CA composite with temperature under different pressures.

pleted. Under all conditions, the weight loss fraction increases with increasing temperature. At $1073 \mathrm{~K}$, the weight loss fraction of the composite under all conditions is almost $40 \%$. However, the temperature at which the weight loss fraction starts to increase under higher pressure is lower than that under atmospheric pressure. This behavior is caused by the acceleration of the reduction reaction ${ }^{12)}$. An increasing trend in the weight loss fraction is observed with increasing total pressure.

The changes in the density of the composite with temperature under high pressures are also shown in Fig. 4. The change in the density of the composite under high pressure is similar to the trend observed under atmospheric pressure. However, the initial temperature of this change is lower. Furthermore, the density increases after showing a minimum value. In this study, the minimum density was obtained at $1273 \mathrm{~K}$ under $0.3 \mathrm{MPa}$, and the weight loss fraction, porosity, and volume change ratio were $98.1 \%, 97.7 \%$, and $507 \%$, 
respectively. The volume change ratio was calculated from the following equation,

$$
(\text { Volume change ratio })=\frac{V_{2}}{V_{1}} \times 100
$$

where V1 and V2 are the volume of composite sample before and after experiment, respectively. The fractured surface of the CA composite heated to $1273 \mathrm{~K}$ under $0.3 \mathrm{MPa}$, which shows the minimum density, is shown in Fig. 6. After the experiment, the CA composite samples could be easily cut by a wooden toothpick. Many iron whiskers with a diameter of approximately $1 \mu \mathrm{m}$ and a large aspect ratio are observed. Large pores with the size of approximately $100 \mu \mathrm{m}$ are observed from Fig. 12(a). These pores were originated from the space of charcoal, and charcoal disappeared by the gasification reaction. Figure 7 shows the microstructure of the CA composite heated to $1251 \mathrm{~K}$ under atmospheric pressure with a weight loss fraction of $65.0 \%$, and the microstructure of the CA composite heated to $1223 \mathrm{~K}$ under 1.0 MPa with a weight loss fraction of $74.7 \%$. The grey and white areas observed are $\mathrm{FeO}$ and metallic iron, respectively. The thin rod with dark grey indicated by the arrow is charcoal that remained after the completion of the reaction. $\mathrm{FeO}$ is several dozen micrometers in size, while the initial size of the hematite is $0.5 \mu \mathrm{m}$. This indicates that the particles of the raw materials are sintered during the reduction reaction. Under both high and atmospheric pressures, the whisker formation of the metallic iron on the FeO particle is observed. Then, this iron whisker formation leads to the expansion of the composite.

\subsection{Effect of charcoal characteristics on swelling of composite}

Using charcoal A, the swelling behavior of the composite was observed. On the contrary, the composite containing coal and graphite shrunk after the experiment, as described above in Fig. 3. In order to discuss the reason of the difference between $\mathrm{CA}$ and coal, other types of charcoal, namely $\mathrm{CB}$ and $\mathrm{CC}$, were investigated. The appearance of the composites after being heated up to $1273 \mathrm{~K}$ under $0.3 \mathrm{MPa}$ is shown in Fig. 8. The composites obtained by using the three types of charcoal have different appearances. Table 3 lists the volume change ratio and the weight loss fraction of the composites. Maximum volume change ratio was observed for the CA composite. On the other hand, the CB composites shrink. The volume change ratio of the $\mathrm{CB}$ composite shows the minimum value. The difference of shape change of the composites is the same as the difference between the amounts of the volatile matter in charcoals. The weight loss

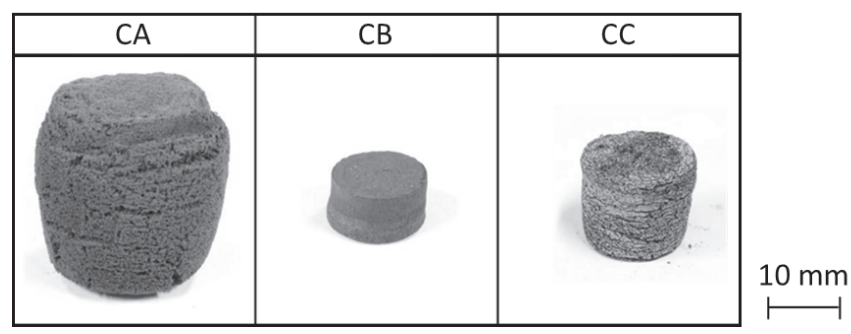

Fig. 8 Appearances of composite samples using $\mathrm{CA}, \mathrm{CB}$, and $\mathrm{CC}$ of charcoal, heated up to $1273 \mathrm{~K}$ under $0.3 \mathrm{MPa}$. (a) Low magnification

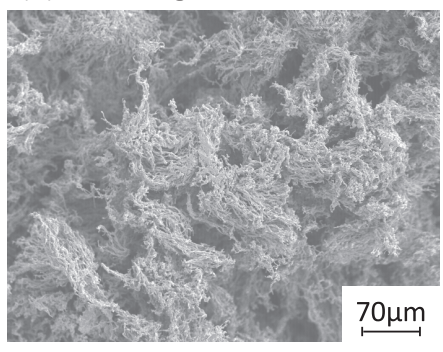

(b) Middle magnification

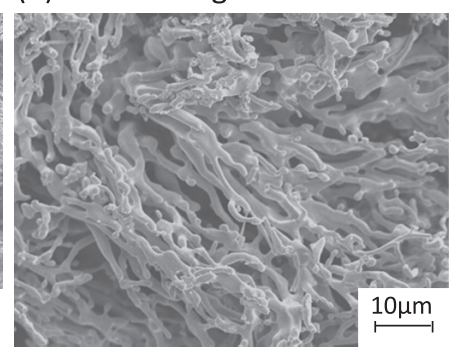

(c) High magnification

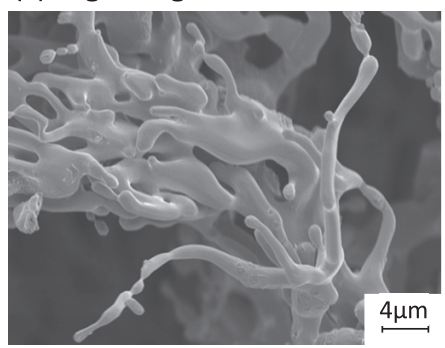

Fig. 6 SEM images of CA composites heated up to $1273 \mathrm{~K}$ under $0.3 \mathrm{MPa}$. (a) low, (b) middle, and (c) high magnifications.

(a)

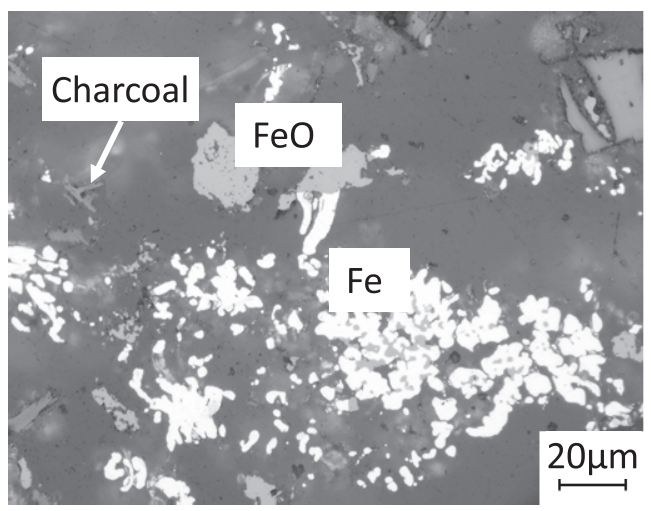

(b)

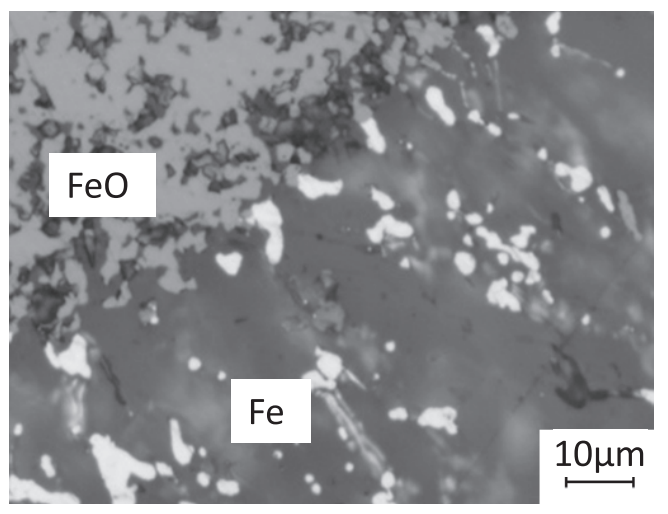

Fig. 7 Microstructures of CA composites heated up to (a) $1251 \mathrm{~K}$ under $0.1 \mathrm{MPa}$ and (b) $1223 \mathrm{~K}$ under atmospheric pressure. 
fraction of the $\mathrm{CA}$ and $\mathrm{CB}$ composites after heating up to $1273 \mathrm{~K}$ are above $90 \%$, indicating that the reduction reaction is almost completed. However, that of the $\mathrm{CC}$ composite is $67 \%$ showing that the metallization degree of $\mathrm{CC}$ is less than $50 \%$. SEM images of the fracture surface of $\mathrm{CB}$ and $\mathrm{CC}$ composites are shown in Fig. 9. The observed phase of the $\mathrm{CB}$ composite is metallic iron because the reduction reaction is almost complete; however, it is not whisker but plate-like in structure. In the case of the CC composite, the dark grey area on the left side of the photo is $\mathrm{FeO}$. The shape of the formed metallic iron is midway between a whisker and plate. These charcoals have different volatile matter contents and ash compositions.

Figure 10 shows the appearance of the CA and CA-800 composites heated to $1123 \mathrm{~K}$ under $0.3 \mathrm{MPa}$. The volume change ratio and weight loss fraction of these composites are listed in Table 3. CA-800 has different volatile matter content from CA because an additional pre-heat treatment at $1073 \mathrm{~K}$ was carried out. The ash composition and the amount of the volatile matter in the CA- 800 are similar to that of $\mathrm{CB}$. However, the volume change ratio of the CA-800 composite is $225 \%$, which is much higher than that of the $\mathrm{CB}$ composite, even though the temperature is $50 \mathrm{~K}$ lower. However, it shows a similar volume change ratio to that of the CA composite at the same temperature. Hence, it can be concluded that the amount of the volatile matter has a lesser effect on abnormal swelling.

The ash in charcoal is composed of various elements as shown in Table 2. CA has a high concentration of $\mathrm{Na}$ and $\mathrm{S}$, whereas $\mathrm{CB}$ has a high $\mathrm{Mg}$ concentration. To negate the effect of ash, a deashing treatment of CA was carried out, and the resulting ash was described as $\mathrm{CA}^{\prime} . \mathrm{NaOH}$ was added to $\mathrm{CA}^{\prime}$, and the obtained ash was described as CA'-Na. Figure 11 shows the appearance of the $\mathrm{CA}^{\prime}$ and $\mathrm{CA}^{\prime}-\mathrm{Na}$ composites heated to $1273 \mathrm{~K}$ under $0.3 \mathrm{MPa}$. The volume change ratios and weight loss fractions of these composites are listed in Table 3. The weight loss fraction of the CA' composite is higher than $90 \%$. It indicates that the reduction reaction is almost complete. The volume change ratio is less than $100 \%$, while that of the CA composite is higher than $500 \%$. The microstructures of these composites are shown in Fig. 12. The formed metallic iron is not whisker-shaped and is similar to that of the $\mathrm{CC}$ composite. These results indicate that some elements in ash affect the swelling behavior. The addition of $\mathrm{Na}$ to the $\mathrm{CA}^{\prime}$ composite slightly increased the volume change ratio of the CA'-Na compared to that of the

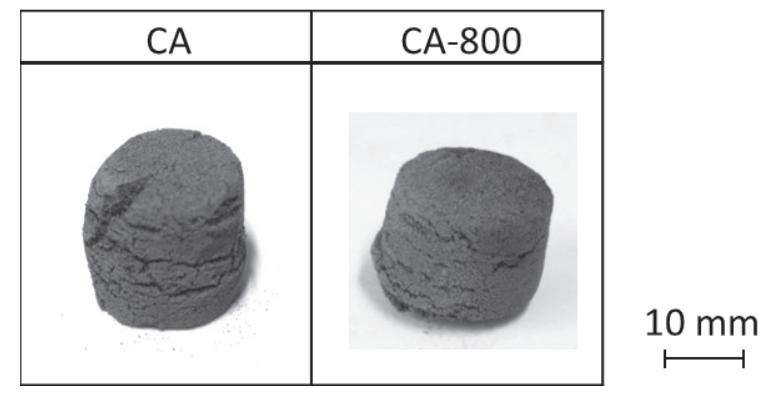

Fig. 10 Appearances of (a) CA and (b) CA-800 composites heated up to $1223 \mathrm{~K}$ under $0.3 \mathrm{MPa}$

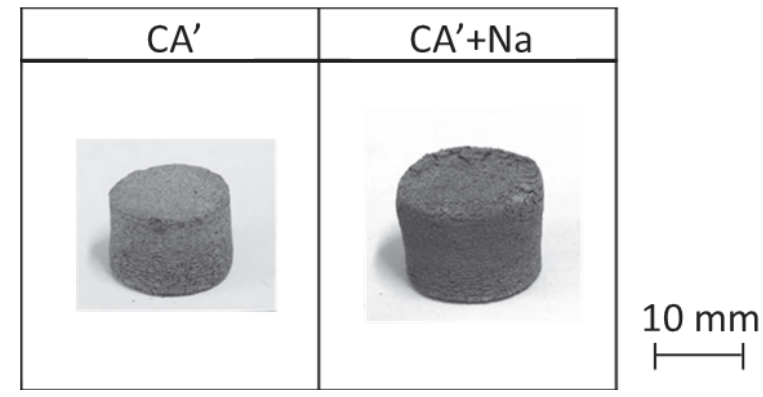

Fig. 11 Appearances of the composite samples using CA' and CA'-Na at $1273 \mathrm{~K}$ under $0.3 \mathrm{MPa}$.

Table 3 Volume change ratio and weight loss fraction of composite obtained using various charcoal.

\begin{tabular}{lcccccc}
\hline Charcoal & CA & CB & CC & CA & CA-800 & CA' \\
\hline Temperature $(\mathrm{K})$ & & 1273 & & 223 & 1273 \\
\hline Volume change ratio (\%) & 507 & 54.5 & 230 & 282 & 225 & 71.0 \\
\hline Weight loss fraction $(\%)$ & 98.1 & 91.2 & 67.0 & 77.3 & 89.1 & 93.9 \\
\hline
\end{tabular}

(a)

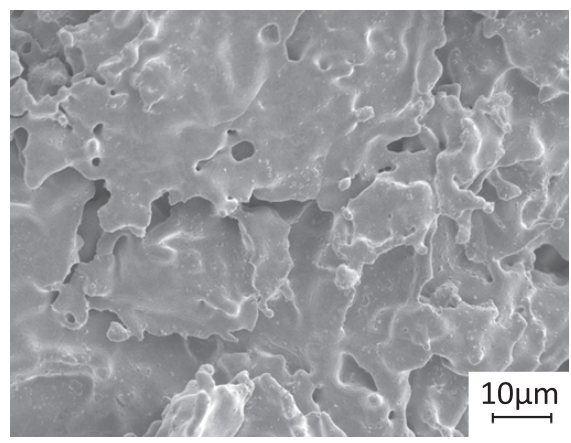

(b)

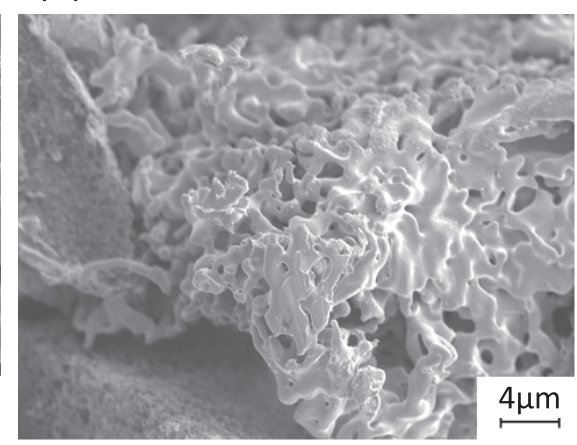

Fig. 9 SEM images of (a) CB and (b) CC composites heated up to $1373 \mathrm{~K}$ under $0.3 \mathrm{MPa}$. 
(a)

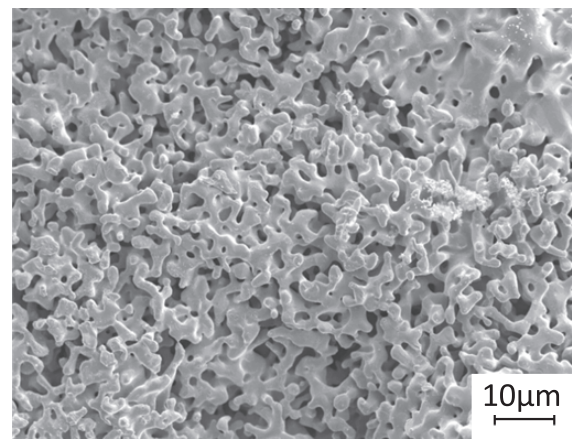

(b)

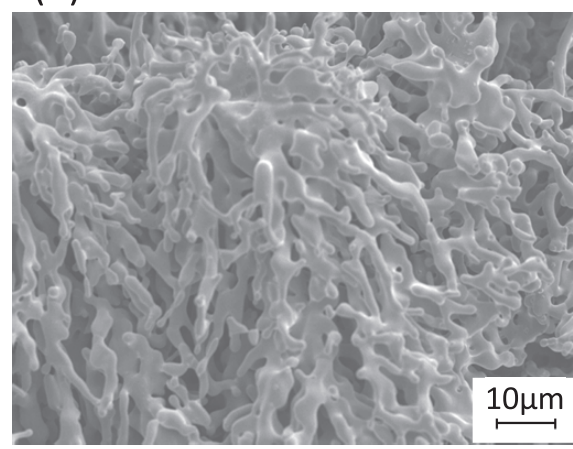

Fig. 12 SEM images of CA' and CA'-Na composites heated up to $1273 \mathrm{~K}$ under $0.3 \mathrm{MPa}$.

CA' composite, while both weight loss fractions were higher than $90 \%$. The shape of the formed metallic iron of the CA'-Na composite looks like whiskers, while the aspect ratio is much smaller than that of the CA composite shown in Fig. 6. It means that $\mathrm{Na}$ affects the formation of iron whiskers. However, its mechanism has not be clearly understood yet. The observed aspect ratio was smaller and abnormal swelling did not occur. It indicates that not only $\mathrm{Na}$ but also other elements such as $\mathrm{K}$ and $\mathrm{S}$ are need to be considered to discuss its mechanism. Furthermore, macro-pores are also observed in these samples as is the case with the CA composite.

\section{Conclusions}

A new production principle of porous iron is proposed by applying the carbothermic reduction reactions with the swelling behavior of composite consisted of a mixed powder of iron oxide and carbonaceous material. The following results are obtained.

(1) The swelling behavior of the composite strongly depends on the type of carbonaceous materials. The composite shrinks during reduction when graphite and coal are used. In contrast, the composite containing charcoal with a higher amount of $\mathrm{Na}$ and $\mathrm{S}$ in the ash shows abnormal swelling, and the formed metallic iron is whisker-shaped with a diameter of approximately $1 \mu \mathrm{m}$ when heated up to $1373 \mathrm{~K}$ under $0.3 \mathrm{MPa}$. The aspect ratio of this whisker is significantly large. The maximum porosity of the obtained composite is $97.7 \%$.

(2) The total pressure affects the swelling behavior because the reduction temperature decreases with increasing pressure. It indicates that this swelling is closely related to the reduction reaction. Furthermore, sintering of the formed metallic iron occurs during reduction. Therefore, the balance between the swelling and sintering leads to the resultant porosity.

(3) When using deashed charcoal, the swelling by the formation of whisker-shaped iron in the composite was not observed. In the composite obtained by the adding $\mathrm{Na}$ to the deashing charcoal, however, whisker-shaped iron formed, but significant swelling did not occur. Therefore, it can be concluded that the components of ash are important factors to control the swelling behaviors.

\section{REFERENCES}

1) H. Nakajima: Ferrum 16 (2011) 599-606.

2) J. Banhart: Prog. Mater. Sci. 46 (2001) 559-632.

3) T. Murakami and E. Kasai: Bulletin of the Advanced Materials Processing Building 62 (2007) 37-45.

4) C. Park and S.R. Nutt: Mater. Sci. Eng. A 288 (2000) 111-118.

5) T. Murakami, K. Ohara, T. Narushima and C. Ouchi: Mater. Trans. 11 (2007) 2937-2944.

6) T. Shimizu and K. Matsuzaki, Proc. Int. Conf. on Porous metals and Metal Foaming Technology, ed. by H. Nakajima and N. Kanetake (The Japan Inst. Metals, 2005) pp. 191-194.

7) S. Ghouse, S. Babu, R.J. Van Arkel, K. Nai, P.A. Hooper and J.R.T. Feffers: Mater. Des. 131 (2017) 498-508.

8) T. Matsumura, Y. Takenaka and M. Shimizu: Tetsu-to-Hagané 85 (1999) 652-657.

9) S. Watanabe and M. Yoshinaga: Sumitomo metals, 17, (1965) 323-327.

10) Y. Fuwa and S. Banya: Tetsu-to-Hagané 52 (1966) 231-233.

11) Z. Qi, T. Murakami and E. Kasai: ISIJ Int. 52 (2012) 1777-1783.

12) T. Murakami, T. Nishimura and E. Kasai: ISIJ Int. 49 (2009) 1686-1693.

13) T. Murakami, T. Nishimura, N. Tsuda and E. Kasai: ISIJ Int. 53 (2013) 1763-1769. 